\title{
MULTIMORBIDADE EM TRABALHADORES AÇOUGUEIROS FEIRANTES
}

\section{MULTIMORBITY IN BUTCHERWORKERS}

\section{MULTIMORBIDAD EN TRABAJADORES CARNICEROS AMBULANTES}

Vaneça da Silva Moreira Magalhães ${ }^{1}$, Ayêsha Alannah Fonseca Mota ${ }^{2}$, Polyana Leal da Silva ${ }^{3}$, Dieslley Amorim de Souza ${ }^{4}$, Mauro César Ribeiro dos Santos ${ }^{5}$, Marcela Andrade Rios ${ }^{6}$.

\section{RESUMO}

Objetivo: analisar os fatores associados à presença de multimorbidade em açougueiros informais de um mercado municipal. Métodos: estudo censitário, de corte transversal, baseado em dados das características sociodemográficas, ocupacionais, de estilo de vida e das doenças e agravos dos trabalhadores açougueiros de um Mercado Municipal. Os dados foram analisados, por meio do Epi Info versão 7.0, com cálculos de razão de prevalência e teste do qui-quadrado e exato de Fisher, adotando-se valor de $\mathrm{p}<0,05$ como estatisticamente significante. Resultados: foram entrevistados 97 açougueiros, com prevalência de 48,5\% de multimorbidade. Foi possível verificar que as variáveis que tiveram associação independente com a multimorbidade foram: sexo feminino $(p=0,018)$, idade superior a 44 anos $(p=0,001)$, escolaridade até ensino fundamental $(p=0,012)$; tempo no comércio menor que oito anos $(p=0,019)$, baixo índice de capacidade para o trabalho $(p=0,000)$, satisfação com a vida $(p=0,025)$ e estresse $(p=0,010)$. Conclusão: Em virtude da magnitude da multimorbidade e da exposição dos açougueiros informais a diversos fatores associados, destaca-se a necessidade de políticas voltadas, para essa classe de trabalhadores, com vista à redução dos impactos negativos na saúde, melhora da qualidade de vida e qualidade do trabalho.

Descritores: Trabalhadores; Multimorbidade; Saúde do Trabalhador.

\section{ABSTRACT}

Objective: to analyze the factors associated with the presence of multimorbity in informal butchers of a municipal market. Methods: Census-based cross-sectional study based on data on sociodemographic, occupational, lifestyle and disease characteristics of butchers in a Municipal Market. Data were analyzed using the Epi Info software (version 7.0) with estimates of prevalence ratio and chi-square test and Fisher's exact test, adopting $p$ value $p=<0.05$ as statistically significant. Results: a total of 97 butchers were interviewed, with a prevalence of $48.5 \%$ of multimorbity. It was possible to verify that the variables that had an independent association with multimorbity were: female $(p=0.018)$, age over 44 years old $(p=0.001)$, elementary school degree $(p=0.012)$, time in trade less than 8 years $(p=0.019)$, low labor capacity $(p=0.000)$, life satisfaction $(p=0.025)$ and stress $(p=0.010)$. Conclusion: due to the magnitude of multimorbity and the exposure of the informal butchers to several associated factors, the need for policies aimed at this class of workers is highlighted, aiming at reducing negative health impacts, improving quality of life and quality of work.

Descriptors: Workers; Multimorbidity; Occupational Health.

\section{RESUMEN}

Objetivo: Analizar los factores asociados a la presencia de multimorbilidad en carniceros ambulantes de un mercado municipal. Métodos: Estudio censal transversal basado en datos sobre características sociodemográficas, ocupacionales, de estilo de vida y enfermedades de carniceros en un mercado municipal. Los datos fueron analizados a través del Epi Info versión 7.0 con cálculos de razón de prevalencia y prueba del chi-cuadrado y exacto de Fisher, adoptando un valor de $p<0,05$ como estadísticamente significante. Resultados: Fueron entrevistados 97 carniceros, con predominio del 48,5\% de multimorbilidad. Se verificó que las variables que tuvieron asociación independiente con la multimorbidad fueron: sexo femenino $(p=0,018)$, edad superior a 44 años $(p=0,001)$, escolaridad hasta enseñanza fundamental $(p=0,012)$, tiempo en el comercio menor de 8 años $(p=0,155)$, bajo índice de capacidad de trabajo $(p=0.000)$, la satisfacción con la vida $(p=0,025)$ y el estrés $(p=0,010)$. Conclusión: En virtud de la magnitud de la multimorbilidad y de la exposición de los carniceros ambulantes a diversos factores asociados, se destaca la necesidad de políticas dirigidas a esta clase de trabajadores, con miras a la reducción de los impactos negativos en la salud, la mejora de la calidad de vida y la calidad del trabajo.

Descriptores: Trabajadores; Multimorbilidad; Salud Laboral.

${ }^{1}$ Graduada em Enfermagem pela Universidade do Estado da Bahia. ${ }^{2}$ Graduada em Enfermagem pela Universidade do Estado da Bahia. ${ }^{3}$ Graduada em Enfermagem. Pósgraduanda em Enfermagem e Saúde pela Universidade Estadual do Sudoeste da Bahia. ${ }^{4}$ Graduado em Enfermagem. Mestre e Doutorando em Ciências da Saúde pela Universidade Estadual do Sudoeste da Bahia. ${ }^{5}$ Graduado em Fisioterapia pela Escola Bahiana de Medicina e Saúde Pública. Mestre em Ciências Ambientais e Saúde. ${ }^{6}$ Graduada em Enfermagem e Obstetrícia. Mestre em Enfermagem e Saúde. Doutoranda em Enfermagem e Saúde pela Universidade Estadual do Sudoeste da Bahia.

Como citar este artigo:

Magalhães VSM, Mota AAF, Silva PL, et al. Multimorbidade em trabalhadores açougueiros feirantes. Revista de Enfermagem do Centro-Oeste Mineiro. 2019;9:e3238. [Access___]; Available in:__. DOI: 


\section{INTRODUÇÃO}

O trabalho constitui-se em uma atividade essencial à vida do ser humano, por meio do qual é possível adquirir meios de sobrevivência para si e seus familiares, além de proporcionar convívio social. Entretanto a saúde do indivíduo pode ser prejudicada, tendo em vista os processos de trabalho e as condições laborais nas quais os trabalhadores estão inseridos.

Desde o princípio, o trabalho era desenvolvido com o objetivo de produção da vida na relação ser humano-natureza, criando valores de uso para viabilizar a existência humana. Além de ser fonte de renda econômica, o trabalho também compreende a inserção do indivíduo na sociedade, pois, por seu meio torna-se possível a interação com outros sujeitos sociais ${ }^{(1-2)}$. Logo o significado do trabalho perpassa o âmbito econômico, compreendendo, também, interação com a natureza e relações sociais.

Contudo, na sociedade moderna, o trabalho tem provocado sofrimento, adoecimento, desgaste físico e mental ao trabalhador. No que se refere ao setor informal, essa situação pode ser mais complexa, uma vez que os trabalhadores informais comumente não são reconhecidos pelas agências governamentais; nas estatísticas oficiais, eles são incluídos incorretamente, além de não serem amparados pelas leis trabalhistas vigentes ${ }^{(1-3)}$.

Dentre as várias atividades inerentes à economia informal, pode-se mencionar a feira livre, em que o trabalho desenvolvido apresenta uma série de dificuldades aos feirantes, por exemplo, as longas jornadas de trabalho, exposição a condições laborais inadequadas, inexistência de horários pré-estabelecidos e de ambiente adequado para a realização da refeição, além de problemas econômicos em virtude do caráter autônomo da profissão ${ }^{(4)}$.

No contexto da feira livre, dentre outros profissionais, estão os açougueiros que, em geral, comercializam carnes e frangos, cuja atividade laboral consiste em talhar e cortar as mercadorias com a utilização de ferramentas, que podem levar à ocorrência de lesões em mãos e dedos ${ }^{(5)}$ Nesse sentido, observa-se que o trabalhador feirante, sobretudo os açougueiros, estão expostos a condições laborais precárias que podem ser prejudiciais à sua saúde, influenciar na qualidade de vida, de trabalho e levar à incidência e prevalência de multimorbidade.
Assim, conceitua-se multimorbidade como presença de duas ou mais condições crônicas de saúde no mesmo indivíduo. Estudo realizado com trabalhadores, na Dinamarca, aponta que a multimorbidade está relacionada a vários desfechos negativos de saúde, como a diminuição da qualidade de vida, mobilidade, capacidade funcional, além de ocasionar aumentos de hospitalizações, angústia psicológica, mortalidade e uso de serviços de saúde ${ }^{(6-7)}$.

Estudo com dados da Pesquisa Nacional de Saúde (PNS) de 2013 dispõe que 14,4\% dos entrevistados referiram possuir multimorbidade, sendo essa uma condição mais frequente em grupos desfavorecidos, contribuindo, assim, para as desigualdades na saúde ${ }^{(6-8)}$.

Nessa perspectiva, o presente estudo tem por objetivo analisar os fatores associados à presença de multimorbidade em açougueiros informais de um mercado municipal.

O resultado deste estudo será relevante pela contribuição com a literatura, no sentido de fornecer melhor entendimento sobre multimorbidade em açougueiros, fato que contribuirá à fomentação de políticas públicas voltadas para essa classe de trabalhadores, além de auxiliar no desenvolvimento de ações educativas junto aos açougueiros informais.

\section{MÉTODOS}

Trata-se de um estudo censitário, de corte transversal, baseado em dados das características sociodemográficas, ocupacionais, do estilo de vida e das doenças e agravos relatados pelos trabalhadores açougueiros do Mercado Municipal de Guanambi/BA.

A pesquisa foi desenvolvida, no município de Guanambi, localizado na região Sudoeste do Estado da Bahia, situado a $796 \mathrm{~km}$ da capital do estado, Salvador, cuja população estimada, segundo dados do Instituto Brasileiro de Geografia e Estatística (IBGE), para o ano de 2018 , em 84.014 habitantes ${ }^{(9)}$. Sua principal atividade econômica é o comércio, tornando a cidade um polo comercial.

O local de escolha, para a realização do estudo, foi o Mercado Municipal de Guanambi/BA, local que aglomera grande parte dos trabalhadores feirantes informais do município, que comercializam grande variedade de produtos. Entre os feirantes, encontram-se os trabalhadores do segmento de carnes, seja ela bovina, suína, ovinos ou peixaria, alocados em 85 , distribuídos nos pavilhões dois e três. 
A população foi composta por todos os açougueiros com idade igual ou superior a 14 anos que desenvolviam atividades laborais, no mercado estudado, sem vínculo com a Previdência Social, seja como empregado do açougue ou como autônomo ou proprietário.

Foram adotados critérios de exclusão os açougueiros que desenvolviam suas atividades fora do local especificado pela administração do Centro de Abastecimento; trabalhadores de outras categorias como camelô, verdureiro, ambulantes, hortifruti; trabalhadores açougueiros que possuem vínculo empregatício.

Aqueles trabalhadores que não foram encontrados, após três visitas, em dias diferentes, incluindo uma segunda-feira ou quinta-feira, por serem dias de maior movimento no Mercado, bem como aqueles que não aceitaram assinar o termo de Consentimento Livre e Esclarecido, foram considerados perdas do estudo.

A coleta ocorreu, no período de novembro de 2015 a janeiro de 2016, utilizando-se o formulário QSEST (questionário sociodemográfico, de estilo de vida, trabalho e saúde), elaborado em 1996 e atualizado em 2009 por Monteiro, além de variáveis sobre capacidade para o trabalho, condições de trabalho e de saúde.

Por se tratar de um estudo censitário, inicialmente, foi realizado pelos pesquisadores um levantamento de todos os trabalhadores açougueiros juntamente com a administração do mercado municipal, totalizando 117 trabalhadores.

Foram entrevistados $97 \quad(82,9 \%)$ açougueiros informais, de ambos os sexos, havendo perda de 20 trabalhadores $(17,1 \%)$ por recusa em participar do estudo, ou por não serem encontrados depois das três visitas, em dias de maiores movimentação no mercado. Posteriormente, construiu-se o banco de dados por meio da digitação dos formulários no Microsoft Excel 2010.

As variáveis sociodemográficas estudadas foram: sexo, idade, situação conjugal, escolaridade (até o ensino fundamental e a partir do ensino médio). A idade foi dicotomizada, após cálculo da mediana, em até 44 anos e maiores de 44 anos.

Quanto aos aspectos ocupacionais, foram estudadas as seguintes variáveis: ter outro emprego, tempo de trabalho no comércio, nível de estresse, capacidade para o trabalho e ter sofrido acidentes de trabalho.
O tempo no comércio se refere ao período de tempo expressa em anos, em que o trabalhador desenvolve suas atividades laborais naquele setor da feira. Para a categorização em dois grupos, realizou-se corte na mediana, sendo o primeiro menos de oito anos de trabalho na feira e o segundo a partir de oito anos de trabalho na feira.

O nível de estresse percebido pelos feirantes foi avaliado, por meio do questionamento sobre o estresse, com opções de respostas que variam de 0 (estou totalmente estressado) a 10 pontos (não estou estressado). Em seguida, após o cálculo da mediana, realizouse a dicotomização dos dados, sendo classificado em grupo de trabalhadores que se encontravam estressados (0-7) e trabalhadores que não se encontravam estressados (8-10).

Para a análise da capacidade de trabalho, foi utilizado o Índice de Capacidade para o Trabalho (ICT), instrumento que revela o quão apto está o trabalhador para desenvolver seu trabalho, considerando as exigências físicas e mentais de sua atividade, a condição de saúde do trabalhador e os recursos por ele utilizados. O ICT compreende dez questões distribuídas, em sete dimensões, nas quais cada item recebe uma pontuação que, após a soma, é obtido o valor que varia de 7 a 49 pontos, sendo categorizados em baixo (7-27), moderado (28-36), bom (37-43) e ótimo $(44-49)^{(10)}$. Após realizar o cálculo do ICT, os dados foram dicotomizados em capacidade para o trabalho baixa (7-27 pontos) e capacidade para o trabalho não baixa (28-49).

$\mathrm{Na}$ variável acidente de trabalho, foi considerada a ocorrência de acidentes de trabalho típicos, nos últimos 12 meses, não sendo considerada, para este estudo, a quantidade de acidentes, tipo de lesão e região do corpo afetada, nem os acidentes de trajeto.

No que se refere ao estilo de vida, foi analisada a prática de atividades físicas e uso de tabaco. Quanto ao uso de tabaco, estimou-se o hábito de fumar no momento em que os participantes foram entrevistados. Destaca-se que as variáveis mencionadas foram categorizadas em sim e não.

Além disso, foram analisadas as variáveis satisfação com a vida e satisfação com o trabalho. Os trabalhadores foram questionados o quão satisfeitos estavam com o trabalho atual e a vida atual. Posteriormente, foram categorizadas, separadamente, cada variável, em satisfeitos (aqueles que declaram estar "muito satisfeitos" 
ou "satisfeitos") e insatisfeitos (quem declarou "pouco insatisfeito" ou "insatisfeito"), havendo também aqueles que se encontravam "nem satisfeito, nem insatisfeito". Para verificar a associação com a multimorbidade, foram incluídos, na análise, somente trabalhadores que estavam "satisfeitos" ou "insatisfeitos".

Para descrever a presença de multimorbidade, foi considerado que tivessem diagnóstico médico, a partir de relatos de lesões por acidentes, doenças musculoesqueléticas, doenças cardiovasculares, doenças respiratórias, distúrbios mentais, doença dos órgãos sentidos e/ou neurológica, doenças digestivas, doenças geniturinárias, doenças da pele, doença endócrina e/ou metabólica, doença hematológica e outras.

Os dados foram analisados, por meio do programa estatístico Epi Info ${ }^{\text {Tm}}$, versão 7.0 (Centers for Disease Com-troland Prevention, Atlanta, Estados Unidos), sendo realizada a análise descritiva das variáveis com cálculo de frequência absoluta e relativa. Para verificar os fatores associados à presença de multimorbidade e as características sociodemográficas, bem como ocupacionais e estilo de vida, foi utilizado o teste do Qui-quadrado e Exato de Fisher, adotando-se o valor de $p<0,05$ como estatisticamente significante. Realizou-se, ainda, a estimativa da razão de prevalência (RP) para cada variável estudada.
A variável dependente foi presença de multimorbidade (relato de duas ou mais condições crônicas de saúde) e as independentes aquelas relacionadas aos aspectos sociodemográficos, ocupacionais e de estilo de vida.

Quanto aos aspectos éticos, o estudo foi apreciado e aprovado pelo Comitê de Ética em Pesquisa da Universidade do Estado da Bahia, sob Certificado de Apresentação para Apreciação Ética-CAAE 44126515.5.0000.0057, com o número de parecer: 1.253.270.

\section{RESULTADO E DISCUSSÃO}

Foram entrevistados 97 açougueiros informais que desempenham suas atividades laborais no Mercado Municipal de Guanambi/BA, dentre eles, 47 pessoas apresentaram multimorbidade, equivalente a $48,5 \%$ da população estudada. Destaca-se que foram considerados, neste estudo, agravos e doenças crônicas com diagnóstico médico.

Na população estudada, de maneira geral, houve predomínio do sexo masculino (67,0 \%), casados $(73,2 \%)$, na faixa etária de até 44 anos $(50,5 \%)$, com grau de escolaridade acima do ensino médio $(55,7 \%)$ e tempo de trabalho mais de oito anos no comércio (51,5\%), conforme visualizado na Tabela 1.

Tabela 1 - Características da população do estudo, segundo variáveis sociodemográficas, ocupacionais e relacionadas ao estilo de vida. Guanambi, Bahia, Brasil, 2018.

\begin{tabular}{|c|c|c|c|c|}
\hline \multirow{2}{*}{ Variáveis } & \multicolumn{2}{|c|}{ População total do estudo } & \multicolumn{2}{|c|}{ Multimorbidade } \\
\hline & $n$ & $\%$ & $\mathbf{n}$ & $\%$ \\
\hline \multicolumn{5}{|l|}{ Sexo } \\
\hline Feminino & 32 & 33,0 & 21 & 44,7 \\
\hline Masculino & 65 & 67,0 & 26 & 55,3 \\
\hline \multicolumn{5}{|l|}{ Idade } \\
\hline Até 44 anos & 49 & 50,5 & 16 & 34 \\
\hline Mais que 44 anos & 48 & 49,5 & 31 & 66 \\
\hline \multicolumn{5}{|l|}{ Estado conjugal } \\
\hline Casados & 71 & 73,2 & 34 & 72,3 \\
\hline Não casados & 26 & 26,8 & 13 & 27,7 \\
\hline \multicolumn{5}{|l|}{ Escolaridade } \\
\hline Até o ensino fundamental & 43 & 44,3 & 27 & 57,5 \\
\hline A partir do ensino médio & 54 & 55,7 & 20 & 42,5 \\
\hline \multicolumn{5}{|l|}{ Tempo de comércio na feira } \\
\hline Menos de 8 anos & 47 & 48,5 & 17 & 36,2 \\
\hline A partir de 8 anos & 50 & 51,5 & 30 & 63,8 \\
\hline \multicolumn{5}{|l|}{ Ter outro trabalho } \\
\hline Sim & 13 & 13,4 & 7 & 14,9 \\
\hline Não & 84 & 86,6 & 40 & 85,1 \\
\hline \multicolumn{5}{|l|}{ Capacidade para o trabalho } \\
\hline Baixa & 13 & 13,4 & 12 & 25,2 \\
\hline Não baixa & 84 & 86,6 & 35 & 74,5 \\
\hline
\end{tabular}




\begin{tabular}{|c|c|c|c|c|}
\hline \multirow{2}{*}{ Variáveis } & \multicolumn{2}{|c|}{ População total do estudo } & \multicolumn{2}{|c|}{ Multimorbidade } \\
\hline & n & $\%$ & $\mathbf{n}$ & $\%$ \\
\hline \multicolumn{5}{|l|}{ Classificação do estresse } \\
\hline Sim & 51 & 52,6 & 31 & 66 \\
\hline Não & 46 & 47,4 & 16 & 34 \\
\hline \multicolumn{5}{|l|}{ Satisfação com o trabalho } \\
\hline Satisfeito & 77 & 79,4 & 33 & 70,2 \\
\hline Insatisfeito & 15 & 15,5 & 10 & 21,3 \\
\hline Nem satisfeito, nem insatisfeito & 5 & 5,2 & 4 & 8,5 \\
\hline \multicolumn{5}{|l|}{ Satisfação com a vida } \\
\hline Satisfeito & 75 & 77,3 & 33 & 70,2 \\
\hline Insatisfeito & 16 & 16,5 & 12 & 25,5 \\
\hline Nem satisfeito, nem insatisfeito & 6 & 6,2 & 2 & 4,3 \\
\hline \multicolumn{5}{|l|}{ Ter sofrido acidente de trabalho } \\
\hline Sim & 60 & 61,9 & 31 & 66 \\
\hline Não & 37 & 38,1 & 16 & 34 \\
\hline \multicolumn{5}{|l|}{ Realiza atividade física } \\
\hline Sim & 41 & 42,3 & 20 & 42,5 \\
\hline Não & 56 & 57,7 & 27 & 57,5 \\
\hline \multicolumn{5}{|l|}{ Fuma } \\
\hline $\operatorname{Sim}$ & 10 & 10,3 & 4 & 8,5 \\
\hline Não & 87 & 89,7 & 43 & 91,5 \\
\hline
\end{tabular}

Fonte: Banco de dados do projeto de pesquisa Condições laborais e de saúde de açougueiros feirantes em cidade de médio porte, 2016.

Considerando os trabalhadores com multimorbidade, a maioria eram homens $(55,3 \%)$, com mais de 44 anos (66\%), casados $(72,3 \%)$, com escolaridade até o ensino médio $(57,5 \%)$, que trabalham há mais de oito anos no comércio $(63,8 \%)$, não possuíam outro trabalho $(85,1 \%)$, apresentavam capacidade para 0 trabalho classificada como não baixa $(74,5 \%)$, encontravam-se estressado (74,5\%), satisfeitos com o trabalho $(76,7 \%)$ e satisfeitos com a vida $(73,3 \%)$. Os trabalhadores relataram ter sofrido acidente de trabalho, nos últimos 12 meses (66\%), não possuir o hábito de realizar atividade física $(57,5 \%)$ e não serem tabagistas $(91,5 \%)$.

Percebe-se, neste estudo, que um número expressivo de açougueiros, quase metade da população estudada apresentava multimorbidade. No que se refere aos impactos da multimorbidade para o trabalho e saúde do trabalhador, autores destacam que o aumento da prevalência de doenças crônicas não transmissíveis (DCNT) pode resultar em diminuição da produtividade, absenteísmo, presenteísmo, invalidez, aposentadoria precoce e aumento nos gastos com a saúde ${ }^{(11)}$.

Em concordância, estudo realizado com trabalhadores americanos identificou que 0 número de faltas no trabalho, ao longo de um ano, era proporcional ao número de condições crônicas de saúde, assim sendo, quanto mais condições crônicas, maior o número de dias de trabalho perdidos ${ }^{(12)}$.
Destarte, percebe-se que a ocorrência de multimorbidade acarreta uma série de danos ao trabalhador, além da deterioração da saúde e comprometimento das atividades diárias; ainda, observam-se prejuízos financeiros, uma vez que há diminuição dos dias trabalhados, queda na produção e capacidade para o trabalho diminuída.

No que se refere ao setor informal, essa situação pode ser agravada, pois, de acordo a Organização Internacional do Trabalho (OIT), no âmbito informal, há uma pluralidade de situações que corrobora para condições desfavoráveis ao trabalhador, tais como locais de trabalho indefinidos, condições de trabalho insalubres e perigosas, baixos níveis de qualificação e produtividade do trabalho, rendimentos baixos e irregulares, longas jornadas de trabalho, falta de acesso à informação e tecnologia, dentre outros ${ }^{(3)}$.

Autores apontam que as condições de trabalho na feira podem contribuir, para o surgimento de doenças, alterações emocionais e deficiência de informação sobre hábitos de vida saudáveis $^{(4)}$. Nesse sentido, percebe-se que os trabalhadores informais estão mais expostos ao risco de adoecimento e/ou agravamento de problemas de saúde, em virtude das condições de trabalhos nas quais estão inseridos.

$\mathrm{Na}$ análise do qui-quadrado, foi observada associação entre multimorbidade e as variáveis sexo $(p=0,018)$, idade $(p=0,001)$ e escolaridade $(p=0,012)$, conforme apresentado na Tabela 2. 
As mulheres apresentaram maior prevalência de multimorbidade (64\%) quando comparadas aos homens. Aqueles trabalhadores com idade superior a 44 anos apresentaram (97\%) maior prevalência, quando comparados aos mais jovens, e a escolaridade menor que oito anos (ensino fundamental) também mostrou maior prevalência (69\%) comparada aos de maior escolaridade.

Tabela 2 - Análise inferencial bivariada dos fatores sociodemográficos e valor de $p$, na associação com multimorbidade, em trabalhadores açougueiros informais do comércio. Guanambi, Bahia, Brasil, 2018.

\begin{tabular}{lcccc}
\hline \hline$\quad$ Variáveis sociodemográficas & $\%$ & RP & IC 95\% & $p$ valor* \\
\hline Sexo & & & & \\
Feminino & 65,6 & 1,64 & $1,11-2,42$ & 0,018 \\
Masculino & 40,0 & 1,00 & - & \\
Idade & & & - & \\
Até 44 anos & 32,6 & 1,00 & $1,25-3,11$ & 0,001 \\
Mais que 44 anos & 64,5 & 1,97 & & 0,854 \\
Estado conjugal & & & $0,60-1,50$ & \\
Casados & 47,8 & 0,95 & - & 0,012 \\
Não casados & 50,0 & 1,00 & & \\
Escolaridade & & & $1,11-2,57$ & - \\
Até o ensino fundamental & 62,7 & 1,69 & \\
A partir do ensino médio & 37,0 & 1,00 & &
\end{tabular}

Fonte: Banco de dados do projeto de pesquisa Condições laborais e de saúde de açougueiros feirantes em cidade de médio porte, 2016. IC95\%: intervalo de $95 \%$ de confiança; RP: razão de prevalência. * Obtido pelo teste do qui-quadrado.

Em relação ao sexo, percebe-se que a prevalência foi (64\%) maior nas mulheres, quando comparadas aos homens. Entretanto esse resultado deve ser observado com cautela, pois se questiona a baixa adesão dos homens ao serviço de saúde, tornando difícil o diagnóstico precoce da multimorbidade. Geralmente, os homens dispensam menor atenção com a prevenção e autocuidado e buscam os serviços de saúde já na fase mais avançada da doença ${ }^{(13)}$.

Essa baixa adesão está intimamente relacionada ao conceito de masculinidade estabelecido pela cultura patriarcal, na qual o adoecer é sinônimo de fragilidade, rejeitado pela figura masculina, somado ao sentimento de invulnerabilidade e o seu papel de provedor da família. Outro fator preponderante é a deficiência dos serviços de saúde no que tange às ações voltadas para a saúde do homem, bem como a falta de capacitação profissional para atender esse público ${ }^{(13-14)}$. Logo, esse dado pode estar subestimado pela grande possibilidade de não haver diagnóstico de multimorbidade nesse gênero, tendo em vista que foram considerados, no estudo, somente agravos e doenças crônicas com diagnóstico médico.

Ademais, observa-se o crescente aumento da presença feminina, no mercado de trabalho, sobretudo, no informal, como pode ser evidenciado neste estudo ( $n=32 ; 33 \%)$. Percebese que a mulher ganha espaço, no ramo de açougue, uma profissão em que, culturamente, tem predominância da figura masculina.

Trabalhadores com mais de 44 anos foram mais acometidos ( $\mathrm{RP}=1,97$; IC 95\%: $1,25-3,11$ ) pela multimorbidade quando comparados a indivíduos mais jovens. Pesquisa aponta que, no Brasil, com o avançar da idade, aumenta o número de doenças crônicas não transmissíveis no indivíduo ${ }^{(8)}$. Assim, pode-se afirmar que o envelhecimento consiste em fator de risco para a multimorbidade nos indivíduos, sobretudo, em trabalhadores feirantes.

Além disso, nota-se a permanência de idosos, no mercado de trabalho informal, cuja permanência pode ter origem no sentimento de exclusão vivenciado pelos idosos, fato que dificulta a aceitação em se aposentar, levando-os a permanecerem no mercado de trabalho, principalmente, em atividade que remetem à informalidade. Essa situação pode estar relacionada, também, à forma como a feira livre se organiza e mantém as relações sociais, na qual os feirantes criam suas próprias regras de convivência, mantém relações de ajuda mútua, e a representatividade da feira como um local de 
desenvolvimento e descontração por meio das relações entre feirantes e entre fregueses ${ }^{(15-16)}$.

Estudo realizado com trabalhadores do comércio informal, no município de Jequié, aponta que a maioria dos idosos (66,2\%) informou não possuir o desejo de sair da informalidade, provavelmente, por ser o proprietário do comércio e pela oportunidade de alcançar uma renda maior, comparado a outro emprego ${ }^{(15)}$. Assim, no comércio informal, o trabalhador possui flexibilidade de horários e autonomia no desenvolvimento de suas atividades laborais, o que não seria possível no setor formal.

Outro aspecto sociodemográfico que apresentou associação com multimorbidade foi o nível de escolaridade, em que trabalhadores que estudaram até o ensino fundamental apresentaram maior prevalência (69\%) de multimorbidade, quando comparados àqueles que possuem ensino médio ou mais. Acredita-se que esse dado esteja relacionado ao acesso a informações sobre a importância da adoção de hábitos saudáveis para a prevenção de agravos de saúde e DCNT.

Sobre este aspecto, autores apontam o predomínio de fatores de risco, em indivíduos com até oito anos de estudo, na população de Campinas-SP, a exemplo de tabagismo; menor consumo de frutas, legumes e verduras; menor frequência de realização de atividade física; e maior excesso de peso e ingestão de carne com excesso de gordura. Além disso, a maior escolaridade está relacionada à procura por serviços de saúde na presença de algum sintoma ${ }^{(17-18)}$. Nesse sentido, a menor escolaridade associa-se a comportamentos de risco, de igual modo, pessoas com maior grau de instrução têm mais possibilidade de adoção de hábitos saudáveis.

Em relação aos aspectos ocupacionais e de estilo de vida, verificou-se associação com multimorbidade nas seguintes variáveis: tempo no comércio menor que oito anos $(p=0,019)$, capacidade para o trabalho $(p=0,000)$, satisfação com a vida $(p=0,025)$ e estresse $(p=0,010)$, conforme visualizado na Tabela 3.

Tabela 3 - Análise inferencial bivariada das variáveis ocupacionais e estilo de vida e valor de p, na associação com multimorbidade em trabalhadores açougueiros informais do comércio. Guanambi, Bahia, Brasil, 2018.

\begin{tabular}{|c|c|c|c|c|}
\hline Variáveis ocupacionais e de estilo de vida & \% & RP & IC 95\% & $p$ valor* \\
\hline \multicolumn{5}{|l|}{ Tempo de comércio na feira } \\
\hline Menos de 8 anos & 36,1 & 0,60 & $0,38-0,93$ & 0,019 \\
\hline A partir de 8 anos & 60,0 & 1,00 & - & \\
\hline \multicolumn{5}{|l|}{ Ter outro trabalho } \\
\hline $\operatorname{Sim}$ & 53,8 & 1,13 & $0,65-1,96$ & 0,677 \\
\hline Não & 47,6 & 1,00 & - & \\
\hline \multicolumn{5}{|l|}{ Capacidade para o trabalho } \\
\hline Baixa & 92,3 & 2,21 & $1,64-2,98$ & 0,000 \\
\hline Não baixa & 41,6 & 1,00 & - & \\
\hline \multicolumn{5}{|l|}{ Satisfação com o trabalho } \\
\hline Satisfeito & 42,8 & 0,64 & $0,41-0,99$ & 0,092 \\
\hline Insatisfeito & 66,6 & 1,00 & - & \\
\hline \multicolumn{5}{|l|}{ Satisfação com a vida } \\
\hline Satisfeito & 44,0 & 0,58 & $0,40-0,85$ & 0,025 \\
\hline Insatisfeito & 75,0 & 1,00 & - & \\
\hline \multicolumn{5}{|l|}{ Classificação do estresse } \\
\hline Sim & 60,7 & 1,74 & $1,11-2,74$ & 0,010 \\
\hline Não & 34,7 & 1,00 & - & \\
\hline \multicolumn{5}{|l|}{ Ter sofrido acidente de trabalho } \\
\hline $\operatorname{Sim}$ & 51,6 & 1,19 & $0,76-1,86$ & 0,422 \\
\hline Não & 43,2 & 1,00 & - & \\
\hline \multicolumn{5}{|l|}{ Realiza atividade física } \\
\hline Sim & 48,8 & 1,01 & $0,66-1,53$ & 0,956 \\
\hline Não & 48,2 & 1,00 & - & \\
\hline \multicolumn{5}{|l|}{ Fuma } \\
\hline Sim & 40,0 & 0,80 & $0,36-1,78$ & 0,410 \\
\hline Não & 49,4 & 1,00 & - & \\
\hline
\end{tabular}


Em relação aos aspectos laborais dos açougueiros, os trabalhadores, com menor tempo no comércio, apresentaram menor prevalência de multimorbidade. Por outro lado, quanto maior o tempo de trabalho, maior será a exposição, consequentemente, maior prevalência de adoecimento. Quando associado esse achado à variável idade, percebe-se a simultaneidade de fatores nos trabalhadores feirantes, considerando que o avançar da idade predispõe o surgimento de doenças crônicas, somado ao aumento da permanência na informalidade, como já discutido anteriormente.

Nessa perspectiva, autores afirmam que os riscos ocupacionais interferem no processo saúde-doença, por sua interação no corpo do trabalhador, ocasionando danos e deterioração da saúde com várias manifestações clínicas. Estudo realizado com mulheres feirantes identificou que pelas condições de trabalho, em que estão inseridas, são vulneráveis a determinadas situações, a exemplo de adoecimento $^{(4-19)}$.

$\mathrm{Na}$ variável satisfação com a vida, observou-se que a prevalência de multimorbidade foi menor (42\%), em indivíduos satisfeitos com a vida, quando comparados aos insatisfeitos. $O$ julgamento de satisfação compreende o processo de comparação entre as circunstâncias de vida do indivíduo e um padrão por ele escolhido, sendo a satisfação com a vida um dos critérios de bem-estar subjetivo elevado $^{(20)}$.

Assim sendo, trabalhadores com multimorbidade podem estar insatisfeitos com a vida, em razão da deterioração do corpo e diminuição da qualidade de vida, pois condições crônicas de saúde influenciam negativamente a qualidade de vida, em longo prazo, afeta o desenvolvimento das atividades cotidianas ${ }^{(21)}$. Nessa perspectiva, enfatiza-se a importância do diagnóstico precoce, especialmente das doenças laborais, tendo em vista a promoção da qualidade de vida e melhora da satisfação.

Em relação ao Índice de Capacidade para o Trabalho (ICT), açougueiros que apresentaram baixa capacidade para o trabalho possuem duas vezes mais risco, quando comparados àqueles trabalhadores com capacidade para o trabalho classificada como não baixa, apresentando forte associação $(p=0,000)$. Sobre este aspecto, estudo realizado, na Dinamarca, identificou que a quantidade de doenças crônicas, presentes nos trabalhadores, apresentou associação progressiva à ausência de longa duração e boa capacidade para o trabalho prejudicada ${ }^{(7)}$.

A capacidade para o trabalho é entendida como condição resultante da combinação entre recursos humanos, em relação às demandas físicas, mentais e sociais do trabalho, gerenciamento, cultura organizacional, comunidade e ambiente de trabalho ${ }^{(22)}$. Percebese que o trabalhador com multimorbidade pode ter comprometimento, na execução de sua atividade laboral, bem como prejuízos financeiros.

Trabalhadores açougueiros que se encontravam com nível de estresse significativo apresentaram razão de prevalência de 1,74 (IC 95\%: $1,11 \quad$ - 2,74), com associação estatisticamente significante à multimorbidade.

Por estresse, entende-se como uma resposta fisiológica neuroendócrina que pode ser adaptativa aos estímulos pertinentes ao estilo de vida. $O$ estresse pode ser benéfico, ao estimular o corpo, melhorando sua atuação, no entanto, quando o estresse atinge um nível considerável, surge a fadiga, em longo prazo, ocasiona suscetibilidade a adoecimento físico e mental ${ }^{(23)}$.

Sobre o impacto à saúde do trabalhador, o estresse, no ambiente de trabalho, pode ocasionar transtornos mentais, doenças cardiovasculares, musculoesqueléticas e reprodutivas; problemas comportamentais, incluindo abuso de álcool e drogas, aumento do tabagismo e distúrbios do sono ${ }^{(24)}$. Nesse sentido, o estresse exerce influência na qualidade de vida e saúde do indivíduo podendo ser um fator contributivo para a ocorrência de multimorbidade em trabalhadores.

\section{CONSIDERAÇÕES FINAIS}

É evidente que os açougueiros informais vivenciam situações de vulnerabilidade pelas características laborais da sua profissão e inserção precária no setor informal. No presente estudo, observou-se que a multimorbidade está associada ao sexo, escolaridade, idade, capacidade para o trabalho, estresse, tempo no comércio e satisfação com a vida.

Vale mencionar as limitações do presente estudo, tendo em vista o seu delineamento transversal, fato que possibilita apenas uma visão instantânea das associações (características sociodemográficas, ocupacionais e estilo de vida) com a variável dependente (multimorbidade). Além disso, o estudo foi realizado apenas com açougueiros informais que desenvolvem suas 
atividades laborais, no mercado municipal de Guanambi, não sendo incluídos aqueles que desenvolvem suas atividades nas residências ou em ruas da cidade.

Nessa perspectiva, faz-se necessária a realização de estudos longitudinais, para melhor esclarecer as associações encontradas no presente estudo.

Em virtude da magnitude da multimorbidade e da exposição dos açougueiros informais a diversos fatores, destaca-se a necessidade de políticas públicas, voltadas para essa classe de trabalhadores, com vista à redução da multimorbidade, melhora da qualidade de vida e condições de trabalho. Sendo assim, destaca-se a necessidade de atividades de educação em saúde junto a esse público com o objetivo de sensibilizar sobre as medidas de prevenção e controle das doenças crônicas e agravos de saúde.

\section{REFERÊNCIAS}

1. Pignati WA, Maciel RHMO, Rigotto RM. Saúde do trabalhador. In: Rouquayrol MZ. Epidemiologia \& saúde. 8a ed. Rio de Janeiro: Medbook; 2018.

2. Vargas FB. Trabalho, emprego, precariedade: Dimensões conceituais em debate. Cad CRH 2016;29(77):313-31. DOI: $10.1590 / \mathrm{SO103}^{-}$ 49792016000200008

3. Krein JD, Proni MW. Economia informal: Aspectos conceituais e teóricos. Brasilia: OIT; 2010.

4. Magalhães AHR, Parente JRF, Silva MAM, Pereira IH, Vasconcelos MIO, Guimarães RX. Necessidade de saúde das mulheres feirantes: Acesso, vínculo e acolhimento como práticas de integralidade. Rev Gaúcha Enferm. 2016;37(nesp):2016-26. DOI: 10.1590/19831447.2016.esp.2016-0026

5. Rios MA, Nery $A A$, Rios PAA, Casotii CA, Cardoso JP. Fatores associados a acidentes de trabalho envolvendo trabalhadores informais do comércio. Cad Saúde Pública 2015;31(6):11991212. DOI: $10.1590 / 0102-311 \times 00101014$

6. World Health Organization (WHO). Multimorbidity: Technical series on safer primary care. Washington: WHO; 2016.

7. Sundstrup E, Jakobsen MD, Mortensen OS, Andersen LA. Joint association of multimorbidity and work ability with risk of long-term sickness absence: A prospective cohort study with register follow-up. Scand J Work Environ Health 2017;43(2):146-54. DOI: 10.5271/sjweh.3620
8. Theme Filha MM, Souza Junior PRB, Damacena GN, Szwarcwald CL. Prevalência de doenças crônicas não transmissíveis e associação com autoavaliação de saúde: Pesquisa Nacional de Saúde 2013. Rev Bras Epidemiol. 2015;18(supl 2):83-96. DOI: $10.1590 / 1980-5497201500060008$ 9. Instituto Brasileiro de Geografia e estatística (IBGE). Guanambi. Brasília: IBGE; 2018 [citado em 2 dez 2018]. Available in: https://cidades.ibge.gov.br/brasil/ba/guanambi/ panorama

10.Silva CF. Índice de capacidade para o trabalho: Portugal e países africanos de língua oficial portuguesa. Aveiro: Departamento de Educação; 2011.

11. Hyeda A, Costa ESM, Sbardellotto F, Ferreira JCC. A aplicação da arquitetura de informação na gestão dos riscos das doenças crônicas em trabalhadores: Uma análise preliminar. Rev. Bras. Med. Trab. 2016;14(1):29-36. DOI: 10.5327/Z1679443520160414

12.Ward BW. Multiple chronic conditions and labor force outcomes: A population study of U.S. adults. Am. J. Ind. Med. 2015;58(9):943-54. DOI: 10.1002/ajim.22439

13.Brasil. Política Nacional de atenção integral à saúde do homem: Princípios e diretrizes. Brasília: Ministério da Saúde; 2009.

14.Carneiro LMR, Santos MPA, Macena RHM, Vasconcelos TB. Atenção integral à saúde do homem: Um desafio na atenção básica. Rev Bras Promoç Saúde 2016 [citado em 29 nov 2018]; 29(4):554-63. Available in: http://periodicos.unifor.br/RBPS/article/view/53 $\underline{01 / p d f}$

15.Biondo CS, Souza Rosa RS, Rios MA, Nery AA. Fatores associados à permanência dos idosos na informalidade. Rev Enferm UFPE 2017;11(5):2090-7. DOI: 10.5205/reuol.930281402-1-RV.1105sup201714

16.Pereira MP, Alencar CMM. Feira livre de São Felipe-BA: Expressões de transformações e resistências à urbanização. Rev Rural Urbano 2016 [citado em 2 dez 2018]; 1(1):58-66. Available

http://www.revista.ufpe.br/ruralurbano/index.ph $\mathrm{p} /$ ruralurbano/article/view/8

17.Francisco PMSB, Segri NJ, Barros MBA, Malta $D C$. Desigualdades sociodemográficas nos fatores de risco e proteção para doenças crônicas não transmissíveis: Inquérito telefônico em Campinas, São Paulo. Epidemiol Serv Saúde 2015;24(1):7-18. DOI: $10.5123 /$ S1679-49742015000100002 
18. Moura EC, Gomes R, Pereira GMC. Percepções sobre a saúde dos homens numa perspectiva relacional de gênero, Brasil, 2014. Ciênc Saúde Coletiva 2017;22(1):291-300. DOI: 10.1590/141381232017221.17482015

19.Shoji S, Souza NVDO, Farias SNP. Impacto do ambiente laboral no processo saúde doença dos trabalhadores de enfermagem de uma unidade ambulatorial especializada. Rev Min Enferm. 2015;19(1):43-8. DOI: $\quad$ 10.5935/14152762.20150004

20.Silveira PMS, Borgatto AF, Silva KS, Oliveira ESA, Barros MVG, Nahas MV. Criação de uma escala de satisfação com a vida por meio da Teoria da Resposta ao Item. J Bras Psiquiatr. 2015;64(4):272-8. DOI: 10.1590/00472085000000089

21.Saraiva LEF, Medeiros LP, Melo MDM, Tiburcio MP, Costa IKF, Torres GV. Condições crônicas de saúde relacionadas a qualidade de vida de servidores federais. Rev Gaúcha Enferm. 2015;36(2):35-41. DOI: 10.1590/19831447.2015 .02 .46666

22. Martinez MC, Latorre MRDO, Fischer FM. Capacidade para o trabalho: Revisão de literatura. Ciênc Saúde Coletiva 2010;15(1):15531561. DOI: 10.1590/S1413-81232010000700067

23.Pinheiro RSA. Impacto do estresse da vida acadêmica nos estudantes de enfermagem [trabalho de conclusão de curso]. São Luís: Universidade Federal do Maranhão; 2018.

24.Organização Mundial de Saúde (OMS), Organização Pan-americana da Saúde (OPAS). Estresse no ambiente de trabalho cobra preço alto de indivíduos, empregadores e sociedade. Brasília: OPAS/OMS; 2016.

Nota: este estudo faz parte do Trabalho de Conclusão de Curso (TCC) intitulado "Multimorbidade em trabalhadores açougueiros feirantes" para obtenção do título de Bacharel em enfermagem pela Universidade do Estado da Bahia (UNEB), Campus XII.

Recebido em: 19/12/2018

Aprovado em: 21/11/2019

Endereço de correspondência:

Vaneça da Silva Moreira Magalhães

Rua Democrata, 464, Centro.

ZIP CODE: 46445-000 - Carinhanha/BA- Brasil

E-mail: moreira.vaneca@gmail.com 